\title{
Collaboration Intensity and Regional Innovation Efficiency in Germany-A Conditional Efficiency Approach
}

\author{
TOM BROEKEL \\ Institute of Economic and Cultural Geography, Leibniz University of Hanover, Germany
}

\begin{abstract}
Many case studies highlight a positive relationship between regions' innovation performance and the intensity of collaboration among regional organizations. However, few efforts have been made to analyze this relation with quantitative approaches. In addition to a theoretical discussion, the paper presents an empirical investigation on this issue utilizing conditional efficiency analysis and patent co-application data for the Electrics \& Electronics industry in 270 German labor market regions. The results show that the relationship between regions' innovation performance and the intensities of regional as well as inter-regional collaboration take the form of an inverted-U shape. Regions with average regional and inter-regional collaboration intensities are found to outperform those characterized by extremely low, high or unbalanced collaboration behavior.
\end{abstract}

KEY WORDS: Regional innovation efficiency, collaboration intensity, patent co-application, conditional efficiency analysis

\section{Introduction}

Numerous case studies show that intensive collaboration among regional organizations fosters their innovation performance (Saxenian, 1998; Asheim and Isaksen, 2002; Cainelli et al., 2007). It is also well known that extensive regional collaboration can yield low innovation performance if inter-regional linkages are lacking (Camagni, 1991). It can therefore be argued that intensive collaboration promotes innovation activities, while beyond a certain intensity level it rather yields negative effects.

In contrast to the qualitative research on this issue, the quantitative empirical picture is still unclear. The majority of studies suggest a positive relation between collaboration 
intensity and innovative success (see, e.g. Arndt and Sternberg, 2000). However, there are also studies that do not find collaboration to play a conducive role for innovation (see, e.g. Fritsch, 2004; Oerlemans and Meeus, 2005).

The present paper aims to shed more light on this issue by systematizing and discussing the relationship between regions' innovation performance and the intensities of regional as well as inter-regional collaboration. Moreover, the relation is quantitatively analyzed using regional data for 270 German labor market regions and the Electrics \& Electronics industry in 1999-2002. In the empirical investigation, regions' innovation performance is assessed as regional innovation efficiency. Its relation with the collaboration intensities is tested using a conditional non-parametric efficiency approach, which is shown to be particularly appropriate and informative in this context.

The results suggest the existence of an inverted-U shape relationship between regional levels of collaboration intensity and innovation efficiency. Average collaboration intensity and a balance of regional and inter-regional collaboration characterize the best performing regions. In contrast, low and very high intensity levels as well as an unbalanced mix of regional and inter-regional collaboration are more frequently found among less performing regions.

The paper is organized as follows. Section 2 gives an overview of the literature on collaboration and regional innovation activities. In Section 3, the empirical methodology is described in detail. The employed data approximating regional and inter-regional collaboration, regional characteristics and innovation are introduced in Section 4. Section 5 presents the results. Section 6 discusses and concludes the study.

\section{Collaboration and Innovation}

\subsection{The Relationship between Collaboration and Innovation}

Inter-organizational collaboration in the field of research and development (R\&D) is an important supplement to internal R\&D activities. Among others the benefits of collaboration activities are the sharing of risk and costs (Cassiman and Veugelers, 2002), as well as access to complementary knowledge (Teece, 1986). However, this does not mean that collaboration is always beneficial. Establishment and maintenance of collaborative agreements require effort and success is not guaranteed. Many collaborations fail, which often implies lost investments. Free-riding is also a known problem in this respect (Kesteloot and Veugelers, 1995). Hence, whether the benefits of collaboration are realized, depends on the complementarity of the partners' resources, aims and working routines (Cantner and Meder, 2007).

The positive effects associated with collaborating have particularly been recognized in Economic Geography. In this literature, it is commonly argued that geographic proximity makes knowledge exchange more likely as "[s] hort distances literally bring people together, favour information contacts and facilitate the exchange of tacit knowledge" (Boschma, 2005: 69). Accordingly, geographic proximity, which may be highly correlated to other forms of proximity with similar effects, ${ }^{1}$ supports the intensity and success of knowledge sharing among organizations (Boschma, 2005).

\footnotetext{
${ }^{1}$ Boschma (2005) names also organizational, cognitive, institutional and social proximity.
} 
For this reason, it can be expected that firms located in regions characterized by a variety of complementary knowledge sources (e.g. many R\&D-intensive firms, private and public research organizations, support organizations for commercializing innovation, etc.) are more likely to benefit from knowledge spillovers than firms in regions lacking these. However, in order to realize these benefits firms need to interact with the according knowledge sources. These interactions may take various forms like labor mobility, social contacts, sharing of facilities and informal as well as formal collaboration in joint R\&D projects. It can therefore be argued that regions with similar knowledge source endowments but unequal interaction intensities among the regional organizations are likely to vary in their innovation performance. ${ }^{2}$ With respect to collaboration, this means that when accounting for variance in regional knowledge endowment, regions characterized by dense regional collaboration are more likely to show higher innovation performance than regions with lower collaboration intensities (Fritsch, 2004).

However, intensive or "excessive" collaboration among members of a closed group (i.e. regional organizations) can sometimes go hand in hand with very dense networks. Such a situation can emerge at the regional level because individuals (and thereby organizations) have a self-reinforcing tendency to concentrate on their home region for knowledge exchange (Broekel and Binder, 2007). Moreover, social aspects that are correlated with geographic proximity can "supersede the economic imperatives" underlying collaboration (Uzzi, 1997: 59).

If networks become too dense, they include inappropriate and redundant relations (Uzzi, 1996). These represent a waste of resources since establishing and maintaining interorganizational connections is not free. Redundant relations may also be harmful due to the danger of knowledge "leaks", that is, the unintended sharing of knowledge that is key to a firm's future competitiveness (De Bondt et al., 1992). The first hypothesis takes this issue up and can be formulated as follows:

Hypothesis 1: The relationship between regions' innovation performance and the collaboration intensity among regional organizations shows as an inverted-U shape. Regions with low and those with very high collaboration intensities are characterized by lower levels of innovation performance than regions with medium intensities.

Although geographic proximity makes regional collaboration more likely, most organizations are also engaged in inter-regional knowledge networks. As for the intensity of regional collaboration, the intensity of inter-regional collaboration impacts regions' innovation performance as well. In particular, inter-regional collaboration gives firms access to knowledge and competences that might not be present within their region (Meyer-Krahmer, 1985). Given the greater variety of potential collaboration partners located outside a particular region and the lower likelihood of redundant links, hypothesis 2 is put forward as:

Hypothesis 2: Higher intensities of inter-regional collaboration foster regional innovation performance.

\footnotetext{
${ }^{2}$ Regional innovation performance refers to the aggregated innovation performance of all regional organizations.
} 
The intensities of regional and inter-regional collaboration are clearly interrelated. Both are influenced by a particular regional collaboration culture (Cantner and Meder, 2008), and specific regional characteristics such as the presence of multinational organizations (Graf, 2010). Moreover, inter-regional collaboration might be a substitute for lacking opportunities to collaborate regionally (Meyer-Krahmer, 1985). Given limited resources to collaborate, intensive regional collaboration might also come at the expense of inter-regional collaboration.

In the context of this paper, it is however more important that regional and inter-regional collaboration are frequently argued to be complements (Camagni, 1991; Bathelt et al., 2004). This means that for achieving superior innovation performance, organizations need to collaborate both regionally and inter-regionally.

I argue that the complementary character of the two types of collaboration is primarily relevant in extreme situations of very unbalanced regional and inter-regional collaboration, which are described in the following. First, regional organizations might be in a situation of high levels of regional and low levels of inter-regional collaboration, which I will refer to as "excessive regional collaboration". Excessive regional collaboration is closely related to, but not to be mistaken for, a regional lock-in. The latter describes the situation of regional organizations being unable to leave a particular development trajectory, which delivers suboptimal economic results (Grabher, 1993). While excessive regional collaboration can be part of a regional lock-in, it only refers to the state of very intense, that is, excessive, collaboration among regional organizations.

Excessive regional collaboration has clearly negative effects for firms' innovation performance because the "ties that bind" have become "ties that blind" (Grabher, 1993: 24) and the regional networks are "too closed and [...] too rigid" (Isaksen, 2001: 110). In other words, by focusing excessively on regional collaboration, organizations miss technologies and innovations developed outside their region. They also lack access to non-regional ideas, knowledge and skills (Camagni, 1991).

Similar arguments can be put forward for the opposite situation in which the intensity of regional collaboration is low while inter-regional collaboration is excessive, which is called "excessive inter-regional collaboration" in the following. Regions in this situation are likely to show low innovation performance because the strong focus on inter-regional relations can lead to segmentation among regional organizations and a quieting of local buzz (Storper and Venables, 2004). Bathelt et al., (2004) argues similarly: a "cluster which is more or less empty because its important organizations are constantly traveling the world in order to build and maintain an extensive pipeline system [global knowledge networks] will run an obvious risk of becoming less vibrant' (48). Not collaborating regionally implies furthermore that firms do not benefit from geographic proximity and its effects on interacting and learning. This includes lower transport and travel costs, easier development of trust, facilitating of collective learning and the exchange of non-codifiable knowledge (see, e.g. Feldman and Florida, 1994; Bathelt et al., 2004). This leads to the third hypothesis:

Hypothesis 3: Situations of unbalanced geographical collaboration (excessive regional and excessive inter-regional collaboration) induce low regional innovation performance.

A last hypothesis concerns the situation in which both, regional and inter-regional, collaboration are frequent. As pointed out before, high levels of regional collaboration can 
result in low innovation performance. A significant portion of this effect is however related to the missing inter-regional collaboration in this situation. To a lesser extent, the same argument holds for situations of excessive inter-regional collaboration in which regional collaboration is underdeveloped. So what can be expected when both, regional and interregional collaboration, are excessive, that is, when regional organizations collaborate excessively in general? It seems reasonable to expect that issues like free-riding, knowledge leakages and the wrong choice of partners become more relevant as the collaboration intensity increases. However, whether these effects collectively impact regional organizations and thereby become visible at the regional level is less clear. In light of the general positive perception of collaborations' effects on innovation, hypothesis 4 puts forward the following:

Hypothesis 4: Situations of excessive regional and inter-regional collaboration are associated with comparatively higher regional innovation performance.

\subsection{Empirical Evidence in the Literature}

A rich empirical literature analyzes the effects of collaboration and knowledge networks on firms' performance (see, e.g. Powell et al., 1996; Uzzi, 1996). These studies generally find that active collaboration has a stimulating impact. However, very dense networks that relate to lower performance are also found to exist (Uzzi, 1996; Fornahl et al., 2011).

The geographic dimension of collaboration and its effect on firms' innovation activities has been investigated as well. A substantial set of case studies provides qualitative evidence for a positive relationship between the collaboration intensity among regional organizations and regions' innovation performance (see, e.g. Saxenian, 1998; Asheim and Isaksen, 2002). However, regional lock-in situations are also observed for regions with low innovation performance (Grabher, 1993).

Few quantitative empirical approaches exist that simultaneously evaluate or compare multiple regions. For instance, drawing on data for 10 European regions, Arndt and Sternberg (2000) find that collaborating with regional and inter-regional partners is particularly relevant for knowledge-intensive firms. Sternberg (1999) adds to this by showing that small and medium-sized firms in three German regions "profit more from intraregional linkages than large firms do" (538). In contrast, Fritsch (2004) uses data on 11 European regions but finds no "support for the suggestion that collaboration or a relatively pronounced cooperative attitude in a region is conducive to innovation activity" (844). Fritsch and Franke (2004) report similar in their study on three German regions.

In light of this mixed and inconclusive picture, it is the objective of the paper to shed more light on this issue by investigating the relationship between the level of regional collaboration intensity and regions' innovation performance. Moreover, it differentiates between the collaboration intensity among regional organizations and the intensity of inter-regional collaboration. In contrast to the above studies that take a firm-level perspective, I employ a regional approach for the empirical investigation. This implies that innovation performance and the two collaboration intensities are conceptualized as regional phenomena referring to the aggregated activities of regional organizations. 


\section{Conditional Non-parametric Efficiency Analysis}

The innovation performance of regions is commonly evaluated in a knowledge production framework (see, e.g. Griliches, 1979; Jaffe, 1989). In this framework, variables representing knowledge inputs are set into a functional relationship with knowledge outputs generated by regional organizations. On this basis, their innovation performance can be perceived of as the efficiency with which knowledge inputs are transformed into innovative outputs (Fritsch, 2003). Brenner and Broekel (2011) explain the idea of the "regional innovation efficiency" as follows:

[i]n such an approach [regional innovation efficiency], we define the innovation performance of a spatial unit by the contribution of this unit to the innovation efficiency of the innovation generators present in the unit. Empirically we would have to measure the number of innovation generators, mainly the R\&D employees or activities in firms ..., and relate this to the innovation output. $(24-25)^{3}$

Fritsch (2003) argues moreover that such a measure can "be regarded as an indication of the quality, particularly the efficiency and workability of the ... regional, or industry-specific innovation system" (85). In accordance with this approach the number of innovation generators in a region represents the input (e.g. the number of R\&D employees) and the number of innovations they generate the innovative output. Their ratio is defined as a region's innovation efficiency, which is influenced by (regional) factors and characteristics, that is, the regional collaboration intensity.

A number of recent studies apply this conceptualization of regional innovation performance as regional innovation efficiency and estimate the latter empirically (see, e.g. Fritsch, 2004; Fritsch and Slavtchev, 2008). I follow Broekel and Brenner (2007) in using a non-parametric approach. In comparison to parametric approaches (e.g. stochastic frontier analysis), the main advantage of non-parametric efficiency analysis is that it does not assume the existence of a universal (pre-defined) functional relationship between knowledge inputs and innovative output. ${ }^{4}$ As for the precise method, I use the order- $m$ efficiency analysis, which has been introduced by Cazals et al. (2002). It is less sensitive to outliers and statistical noise in the data than deterministic non-parametric approaches (e.g. Data Envelopment Analysis).

The principal idea of the order- $m$ efficiency analysis is that observations are evaluated with respect to a frontier function, which consists of best-practice observations, for instance, observations showing a maximum of output ( $Y$ ) given a certain level of input $(X) .{ }^{5}$ Practically, the efficiency measure of order- $m$ can be computed as follows: $Y_{1}, \ldots, Y_{m}$ are $m$ random observations (regions) drawn from the conditional distribution function of $Y$ given $X \leq x_{0}$, that is, only regions with equal or less inputs than region $\left(x_{0}, y_{0}\right)$ are considered. ${ }^{6}$

\footnotetext{
${ }^{3} \mathrm{~A}$ similar but more general definition can be found in Fritsch (2000: 415).

${ }^{4}$ Other advantages include the simultaneous consideration of multiple output variables and that no distributions have to be specified for the error term as well as for the efficiency estimate. A more detailed discussion can be found in Coelli et al., (1998) and Daraio and Simar (2007).

${ }^{5}$ This corresponds to an output-oriented version, which has been argued to be more appropriate in this context than the input-oriented version (Broekel and Brenner, 2007).

${ }^{6}$ Here $m$ is a trimming parameter defining the sensibility of the estimation with respect to outliers in the data.
} 
The output-oriented order- $m$ efficiency measure $\tilde{\lambda_{m}}\left(x_{0}, y_{0}\right)$ is defined for region $\left(x_{0}, y_{0}\right)$ as

$$
\tilde{\lambda}_{m}\left(x_{0}, y_{0}\right)=\max _{i=1, \ldots, m}\left\{\min _{j, \ldots, q}\left(\frac{Y_{i}^{j}}{y_{0}^{j}}\right)\right\}
$$

with $Y_{i}^{j}\left(y_{0}^{j}\right)$ being the $j$ th component of $Y_{i}$ (of yo, respectively). Following Cazals et al., (2002), the final measure $\left(\hat{\lambda_{m}}\right)$ is then the average of a repeated estimation of this efficiency with random draws of $Y_{m}$ (Monte-Carlo algorithm). Values of $\hat{\lambda_{m}}$ smaller or equal to one imply efficiency and larger values represent inefficiency. In the context of the paper, it indicates by how much a region's innovative output has to increase in order for this region to become best practice (efficient) given its level of knowledge inputs.

Intuitively, this efficiency measure could serve as dependent variable in a regression approach and by this means its relationship to other variables ("external factors" in the following) could be investigated. However, such a two-stage procedure, an efficiency analysis that is followed by a regression, can be methodologically problematic (Simar and Wilson, 2007). Conditional efficiency analyses have therefore been developed (Daraio and Simar, 2007). Besides being methodologically superior to a two-stage approach, they offer an additional advantage. Without imposing ex ante a particular type of functional relationship between efficiency and an external factor, they do not only allow assessing the factor's statistical significance but also show the relation for the factor's entire empirical distribution. This is especially important if potential non-linear relationships are to be explored, which is the case in the context of the present paper.

Two measures are estimated in a conditional efficiency analysis: a conditional and an unconditional. The unconditional measure has been described above. The conditional measure also compares the relation between an observation's outputs to the best practice found among observations with equal or less input. In this case, the comparison is however biased such that the likelihood that an observation is compared to another depends negatively on the difference between their values of one or more external factors.

The central variable in this type of analysis is $Q_{z}$ representing the ratio between conditional and unconditional efficiency. To assess the relation between external factors and the estimated efficiency, their values are related to the according $Q_{z}$ values by means of scatterplots and non-linear regressions (for more details, see Daraio and Simar, 2007). The significance of the observed relation and the error bands for the non-linear regressions are estimated with a bootstrap approach based on a mixed kernel function and a data-driven bandwidth selection procedure developed by De Witte and Kortelainen (2009).

\section{Data on Innovation, R\&D and Collaboration}

\subsection{Patent Applications and R\&D Employment}

It is well known that innovation activities differ strongly between industries (see, e.g. Oerlemans and Meeus, 2005). For this reason, I focus on a single industry: the manufactures of electrical and electronic equipment (ELEC). As common in this type of research, the industry's innovation output is approximated with patent data. Patents are by no means perfect measures of innovation (Griliches, 1990). In ELEC patenting is however 
important and the majority of innovations are patented (Arundel and Kabla, 1998), which makes patent counts a reliable innovation indicator. ${ }^{7}$

For the chosen empirical approach, it is important that an inventor's residence and workplace tend to be located within the same region. This is true for the 270 German labor market regions (Greif and Schmiedl, 2002), which have therefore been chosen as unit of analysis. These regions are defined by the German Institute for Labor and Employment and reflect the spatial dimension of labor mobility in Germany. They also correspond to spatial constraints in firms' search for collaboration partners (Broekel and Binder, 2007) allowing for a meaningful differentiation between regional and inter-regional collaboration.

The German Patent Office published the patent data for the years 2001-2004 in Greif and Schmiedl (2002) and Greif et al., (2006). The patents have been regionalized according to the inventor principle. Applications by public research institutes, for example, universities and research societies (e.g. Max Planck Society), as well as patent applications by private inventors are excluded to correctly approximate the innovation activities of private firms.

$R \& D$ efforts are estimated by $R \& D$ employment data, which are obtained from the German labor market statistic provided by the German Federal Employment Agency. It covers all employees subject to social insurance contribution. The concordance by Broekel (2007) is used for the matching between patents and R\&D personnel classification. It is based on the concordance of Schmoch et al., (2003) and adapts it to the particular data used here (see Table 1). On this basis, the innovative output of ELEC is calculated as the sum of all regionalized patents assigned to this industry by Broekel (2007), which results in a single output measure (PAT). A time lag of two years to the R\&D employment data is moreover assumed.

The R\&D employment of ELEC covers three two-digit NACE ${ }^{8}$ codes (DL30, DL31 and DL32). DL30 is characterized by a great number of zero values (147 out of 270). It is therefore summed with DL31 resulting in two input variables. To construct a meaningful efficiency measure, all regions with zero R\&D employment in both R\&D variables (DL30_31, DL32) are excluded, which leaves 258 valid observations per year. ${ }^{9}$

\subsection{Regional Characteristics}

The literature suggests a wide range of regional characteristics that influence firms' innovation and collaboration activities (see, e.g. Feldman and Florida, 1994), which need to be considered as control variables. Foremost this includes urbanization advantages, which are approximated by population density (POP_DEN). The gross domestic product per capita (GDP) captures the regional demand and the public financial situation. The share of employees with high qualifications (HIGH) is considered to measures the quality of local human capital. It also approximates the presence of other high-tech industries. The data for these variables are obtained from the German Federal Institute for Research on Building.

\footnotetext{
${ }^{7}$ It is acknowledged that patents rather capture inventions than innovations. However, in order to stay consistent with the literature, the term "innovation" is used in the paper.

${ }^{8}$ Nomenclature statistique des activités économiques dans la Communauté européenne, Rev. 1.1.

${ }^{9}$ The existence of innovation generators is a prerequisite for the generation of innovation implying that regions with zero R\&D employment are expected to have zero innovations. Efficiency cannot be meaningfully defined in this case.
} 
Table 1. Definition of the Electrics \& Electronics industry according to Broekel (2007)

\begin{tabular}{cc}
\hline Technological fields $^{\mathrm{a}}$ & NACE industries $^{\mathrm{b}}$ \\
\hline $\begin{array}{c}\text { Time measurement, controls, computing } \\
\text { (TF27), Acoustics, electronic data }\end{array}$ & $\begin{array}{l}\text { Manufacture of office machinery } \\
\text { and computers (DL30), Manufacture } \\
\text { storage (TF28), Nuclear physics } \\
\text { (TF29), Electrical engineering (TF30), }\end{array}$ \\
Electronics, communication technology (TF31) & $\begin{array}{l}\text { apparatus n.e.c. (DL31), Manufacture } \\
\text { of radio, television and }\end{array}$ \\
& communication equipment and apparatus \\
& (DL32) \\
\hline
\end{tabular}

${ }^{\text {a }}$ As defined in Greif and Schmiedl (2002).

${ }^{\mathrm{b}}$ According to NACE.

I consider the location coefficient of service employees (SERV) to account for differences in manufacturing and service dominated regions. The variable SPEC represents the location coefficient of ELEC's employees, which approximates advantages related to industrial agglomeration. Missing precise information on the regional firm size distribution, I calculate the average firm size in ELEC (SIZE). The data used to construct these variables are taken from the German labor market statistics.

I also control for non-collaboration-related inter-regional spillovers by accounting for the geographic mobility of university graduates of engineering (GRAD_ENG) and natural sciences \& math (GRAD_NAT) (see Faggian and McCann, 2006). Following the procedure proposed by Broekel and Brenner (2007), the numbers of graduates are distributed across the regions such that a region's probability to obtain another region's graduates depends positively on its population and negative hyperbolically on the geographic distance between the regions. In addition, a certain share of the graduates is allowed to stay in their university's region. The parameters of the hyperbolic function used for estimating the probabilities are fitted by a maximum likelihood calculation using the empirical findings on the mobility of graduates by Legler et al., (2001), which are presented in Table 2. The graduates of each German university and technical college are obtained from the German Statistical Office. To control for size effects, the distributed graduate counts enter the analysis as ratios of regions' total employment.

Table 2. Graduates' mobility

\begin{tabular}{llll}
\hline Distance & $<50 \mathrm{~km}$ & $50-200 \mathrm{~km}$ & $>200 \mathrm{~km}$ \\
\hline GRAD_ENG (University) & $48.8 \%$ & $29.8 \%$ & $21.4 \%$ \\
GRAD_ENG (Technical college) & $42.3 \%$ & $35.5 \%$ & $22.2 \%$ \\
GRAD_NAT (University) & $61.2 \%$ & $14.9 .9 \%$ & 23.9 \\
GRAD_NAT (Technical college) & $45.4 \%$ & $36.0 \%$ & 18.6 \\
\hline
\end{tabular}

Data based on Legler et al., (2001) but adjusted for inner Germany mobility. 


\subsection{Two Collaboration Intensity Measures}

To approximate collaboration the patent data published by the German Patent Office in the "Patentblatt" are used, which includes data from the German and European patent office. In a common fashion, two organizations are argued to collaborate if they jointly apply for a patent (co-application) within the IPC classes assigned to ELEC (see, e.g. Cantner and Meder, 2007).

Co-application agreements are complex and reasons for firms to engage in patent coapplication are manifold (see on this Hagedoorn, 2003). How much of the actual collaboration activities are captured by patent co-applications is unknown. It is however very likely that the share of patent co-applications represents a lower bound of collaboration activities. Since I consider only one industry in one country, it is further reasonable to assume that the share of collaboration resulting in patent co-applications is nearly constant in all regions. Under this assumption is the co-application intensity a valid approximation of the unobserved collaboration intensity (Cantner and Meder, 2008).

The regional collaboration intensity is estimated as proposed by Cantner and Meder (2008). Their measure aims to capture the regionally aggregated collaboration behavior of organizations controlling for the fact that regions vary in their potential for collaborating. The measure is constructed straightforwardly. First, the general collaboration propensity of ELEC is estimated by dividing the number of co-applications with the total number of patents assigned to this industry (the industry's average collaboration intensity). Next, the number of expected co-applications in a region is calculated by multiplying this propensity with the number of regional patents. The ratio $(I)$ between the empirically observed and expected number of co-applications is made symmetric by transforming it with $(I-1) /(I+1)+1 .{ }^{10}$ The resulting measure $(I)$ reflects the regional collaboration intensity. Values of $I$ below 1 indicate lower and values above 1 higher collaboration intensities than the national average.

The regional collaboration intensity (REGION) is constructed on the basis of collaboration between organizations located within the same region. Inter-regional collaboration intensity (INTER) is indicated by the collaborating organizations being located in different German regions. Unfortunately, international collaboration is not considered because of too many zero values in the resulting measure. ${ }^{11} \mathrm{~A}$ time lag of two years with the R\&D employment data is assumed for these measures because they are based on patent data.

Regional and inter-regional collaboration intensities are weakly correlated $\left(r=0.15^{\star \star *}\right) .{ }^{12}$ Figure 1 shows the contour plot for the two intensities highlighting that the mass of observations is characterized by values around one in REGION as well as in INTER.

The correlation between the two collaboration intensities and the number of ELEC patents is $r=0.10^{\star * *}$ for REGION and $r=0.04$ for INTER. As intended, they are more or less unrelated to the magnitude of regions' innovative output.

In line with the results of Cantner and Meder (2008), East German regions are characterized by lower regional but higher inter-regional collaboration intensities than

\footnotetext{
${ }^{10}$ As before, patents are assigned to regions according to the inventor principle.

${ }^{11}$ Accordingly, I have to assume that inter-regional and inter-national collaboration intensities are positively correlated.

$12_{* * *}{ }^{* *}$ and * denote a significance level of $0.01,0.05$ and 0.1 , respectively.
} 


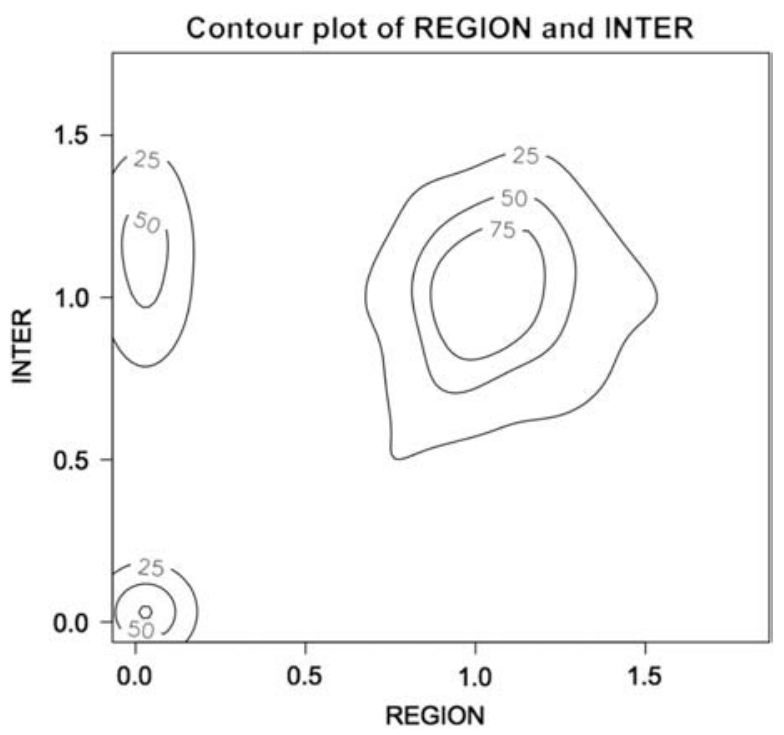

Figure 1. Density of collaboration intensity measures

West German regions. The mean difference for the first is $0.30^{* * *}$ and for the second $\left(0.10^{\star \star \star}\right){ }^{13}$

Table 3 presents some basic descriptions of the variables. Figures 2 and 3 present the corresponding histograms and Table 4 summarizes the correlation structure of all variables considered in the empirical investigation.

\section{Regional Innovation Efficiency and Collaboration Intensities}

\subsection{The Estimation of Regional Innovation Efficiency}

Before testing the relationship between collaboration intensity and regional innovation efficiency, the previously presented control variables are tested for their relevance. For this, I adopt a kind of "stepwise" approach and estimate regions' innovation efficiency considering only R\&D employees as input (EFF). Subsequently, all control variables and combinations of these are tested for their relationship with this measure using the conditional efficiency analysis in analogy to a stepwise regression approach. Eventually, the combination of control variables is chosen that represents the largest set of variables being simultaneously significant. In addition, it is checked if the relationship between each significant control variable and the efficiency estimate is monotone and positive, which is a necessary requirement for a variable to be considered as input in an efficiency analysis (Coelli et al., 1998). ${ }^{14}$

\footnotetext{
${ }^{13}$ Significance is based on Wilcoxon rank sum test.

${ }^{14}$ All estimations are also conducted in two alternative setups. In the first, no control variables are considered and in the second all control variables are taken into account that are significantly correlated to the innovation output. The results are very similar to the reported ones. They can be obtained upon request from the author.
} 
Table 3. Descriptions

\begin{tabular}{lrrrrrr}
\hline VAR & Mean & Std. dev. & Median & Min & Max & Skew \\
\hline PAT & 30.30 & 107.88 & 8.06 & 0.00 & $1,763.33$ & 11.01 \\
DL30_31+DL32 & 656.77 & $1,510.53$ & 196.00 & 0.00 & $16,548.00$ & 6.41 \\
SIZE & 63.00 & 75.40 & 38.70 & 1.67 & 605.83 & 3.69 \\
SERV & 0.09 & 0.23 & 0.05 & 0.01 & 3.93 & 12.89 \\
GRAD_ENG & 0.00 & 0.00 & 0.00 & 0.00 & 0.01 & 1.45 \\
GRAND_NAT & 0.00 & 0.00 & 0.00 & 0.00 & 0.01 & 1.23 \\
GDP & 37.83 & 31.67 & 24.90 & 12.20 & 277.90 & 3.65 \\
HIGH & 11.29 & 10.40 & 7.50 & 2.40 & 83.50 & 3.13 \\
SPEC & 0.94 & 1.05 & 0.56 & 0.01 & 7.43 & 2.58 \\
POP_DEN & 849.71 & $1,277.86$ & 247.00 & 43.00 & $8,495.00$ & 3.03 \\
REGION & 0.69 & 0.58 & 0.84 & 0.00 & 1.86 & 0.00 \\
INTER & 0.882 & 0.44 & 0.98 & 0.00 & 1.86 & -0.67 \\
EFF_H & 943.41 & $4,798.52$ & 2.60 & 0.37 & $53,199.73$ & 6.11 \\
\hline
\end{tabular}

I find that no other control variable gains significance when $\mathrm{HIGH}$ (the share of highly educated employees) is also considered. In addition, none of them undercuts HIGH's $p$-value (see Table 5). It suggests that all control variables explain more or less the same variance in the efficiency measure, which is supported by their comparatively high correlations (see Table 4). HIGH is positively related to innovation efficiency (see Figure 4). Accordingly, the selection criteria are met and $\mathrm{HIGH}$ is considered as input in addition to the two R\&D employment variables.

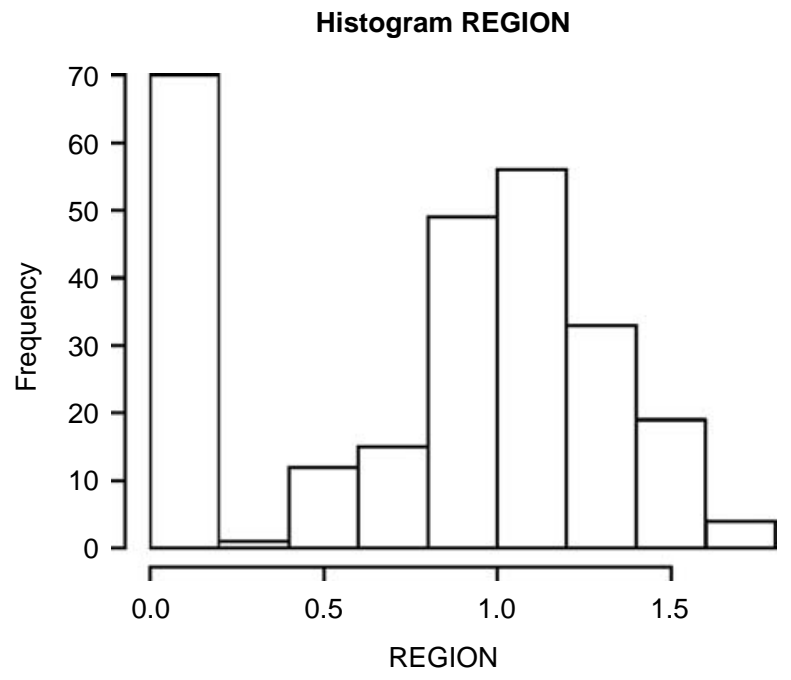

Figure 2. Histogram of REGION 


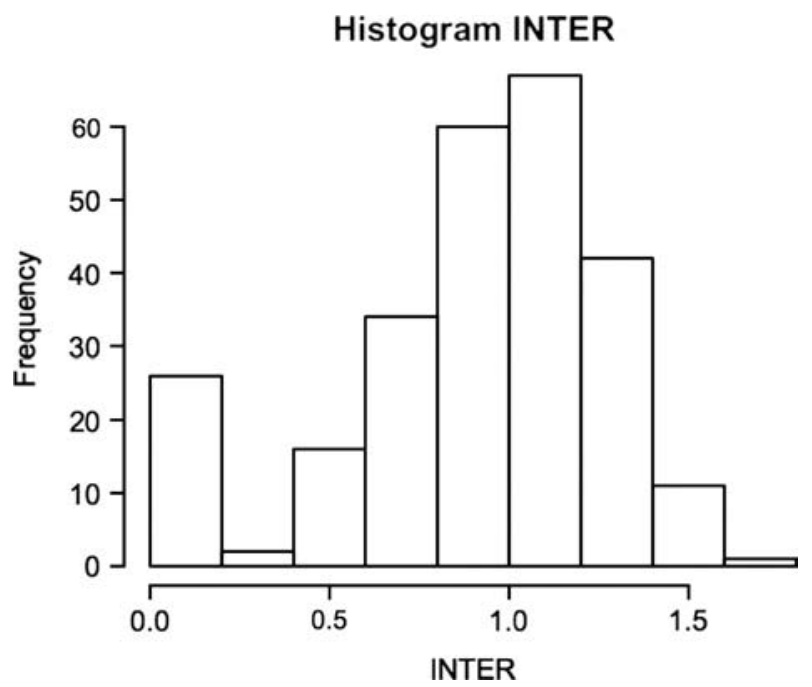

Figure 3. Histogram of INTER

It seems reasonable to assume that the regional level of collaboration intensity is primarily influenced by factors that are more or less time-invariant (i.e. culture, location of region, industrial structure, etc.). This implies that the intensity is subject to little temporal change as well. The available data on R\&D employment covers only four years. For this reason, I argue that any inter-temporal variation in the empirical variables approximating collaboration intensity (within this time span) is most likely statistical noise. This means for the empirical analysis that a panel approach is inappropriate. I therefore estimate the conditional and unconditional efficiency measures separately for each year. Subsequently, the pooled data is used for plotting the scatterplots and drawing the trend curves. ${ }^{15}$ This significantly reduces the effects of outliers and statistical noise and thereby increases the robustness of the results.

\subsection{The Innovation Efficiency of German Regions}

Figure 5 gives an impression of the regional distribution of ELEC's innovation efficiency (EFF_H) considering $\mathrm{HIGH}$ as additional input.

The corresponding histogram is shown in Figure 6 . When looking at the descriptions in Table 3, the first thing to notice is the magnitude of some (in-)efficiency values: EFF_H's mean is 943 while the median is just 5.02. Extremely high efficiency scores are induced by zero output but positive input observations. ${ }^{16}$ Less than 5 per cent of the observations show values of EFF_H $>50$ implying that the distortion is not a minor issue.

\footnotetext{
${ }^{15}$ The ranking of regions according to their efficiency and collaboration intensity values is very stable over time. There are also no indications of significant temporal trends in the variables.

${ }^{16} \mathrm{~A}$ constant of 0.001 is added to all regions' output to ensure proper estimations.
} 


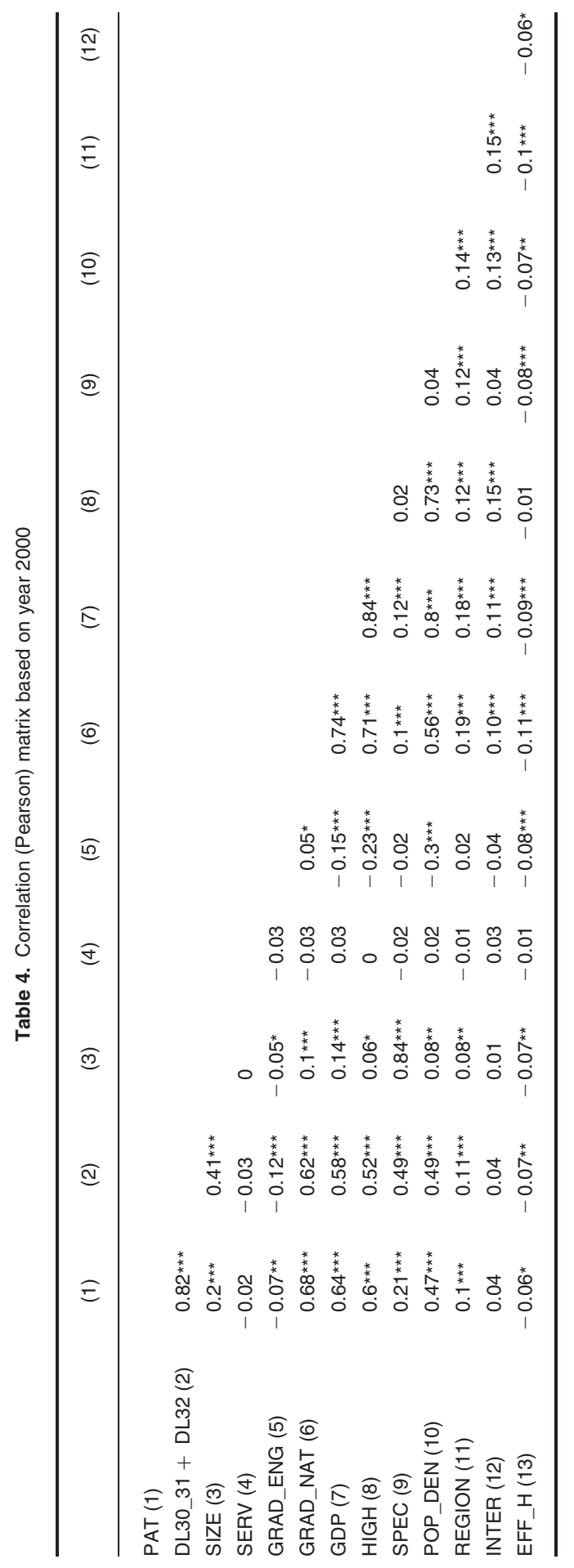


Table 5. $p$-values of variables tested in stepwise procedure

\begin{tabular}{llcr}
\hline 1st variable & p-value & 2nd variable & $p$-value \\
\hline 1. GDP & 0.001 & & \\
2. GDP & 1 & 0.09 & \\
3. HIGH & 0.005 & SIZE & 1 \\
3. HIGH & 0.859 & POP DEN & 1 \\
4. HIGH & 0.901 & SPEC & 0.881 \\
5. HIGH & 0.745 & SERV & 1 \\
6. HIGH & 0.745 & GRAD ENG & 0.341 \\
7. HIGH & 0.001 & GRAD NAT & 0.248 \\
8. HIGH & 0.004 & & \\
\hline
\end{tabular}

\section{Input: R\&D}

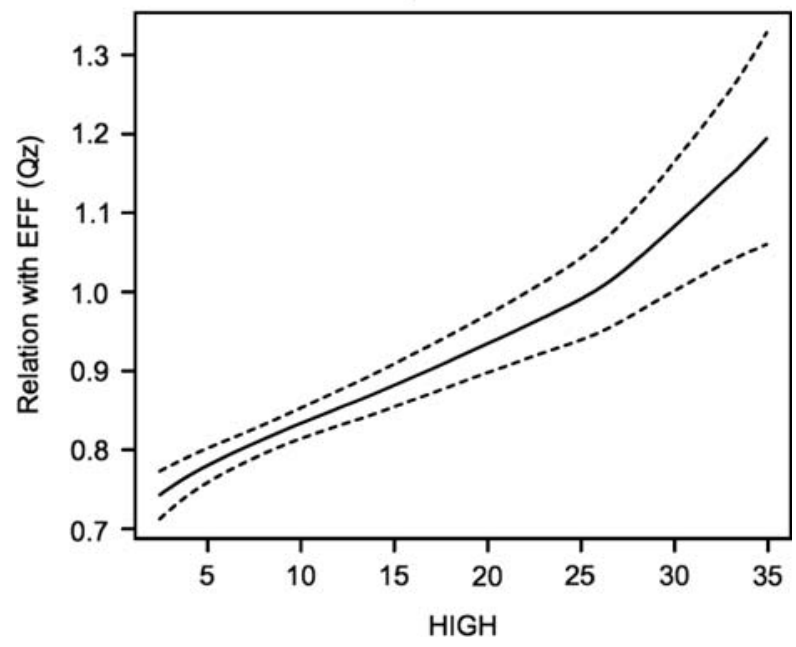

Figure 4. Relation between EFF and $\mathrm{HIGH}$

Supporting the findings by Fritsch and Slavtchev (2008), West German regions are found to be on average more efficient than East German regions (median difference of $\left(11.85^{\star \star \star}\right){ }^{17}$

The geographic distribution (Figure 6) suggests that the absolute number of regional patents is related to the efficiency distribution. While the (Pearson) correlation between EFF_H and total patent output is just $r=0.06^{*}$, the (Spearman) rank correlation confirms this by being as high as $r_{\mathrm{s}}=0.79^{* * *}$. Accordingly, the efficiency analysis significantly impacts the distances between regions in terms of performance but has little effect on the

\footnotetext{
${ }^{17}$ While the efficiency estimate is spatially autocorrelated, the correlation is low in magnitude (Moran's $l: 0.06{ }^{\star \star}$, Geary C: 1.05).
} 


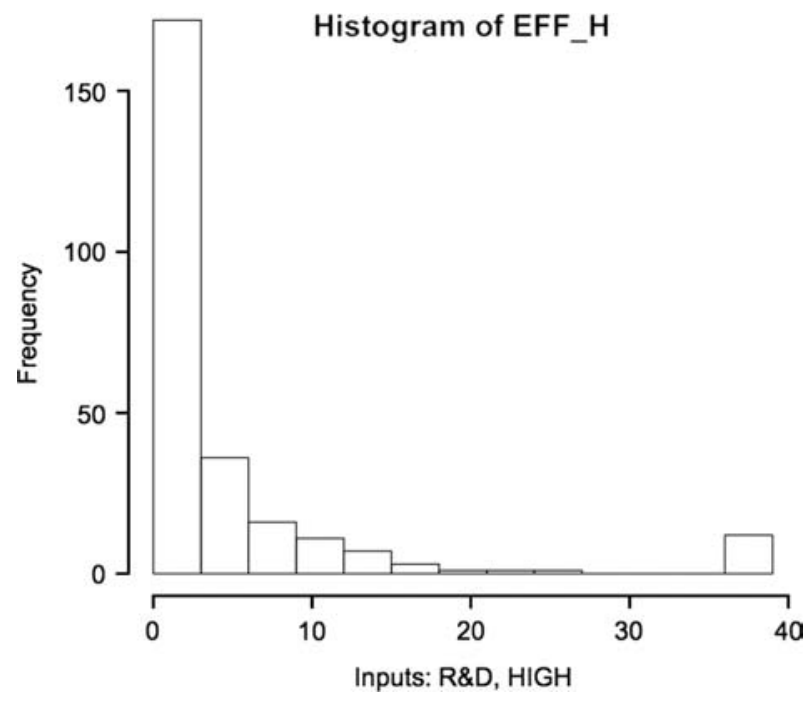

Figure 5. Histogram of EFF_H

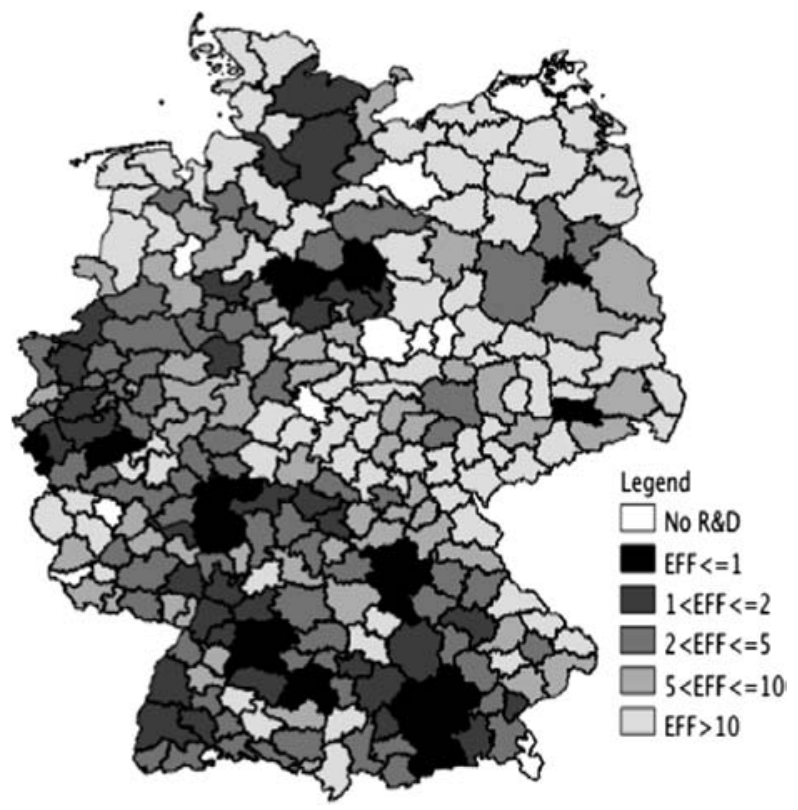

Figure 6. Efficiencies of German labor market regions for ELEC in 2000 
ranking. There are however important exceptions. For instance, Erlangen is ranked 4th in terms of patent output in 2001, while in efficiency it ranks 20th. Bad Tolz is ranked 6th in efficiency but only 26 th in total patent output. ${ }^{18}$

It has to be pointed out though that the correlation between patent numbers and efficiency is unlikely to bias the analysis of the relationship between the latter and collaboration intensity. The reason for this is the number of regional patents being taken into account in the estimation of the collaboration intensity, which results in a low correlation between these two (see Section 4.3).

\subsection{Collaboration Intensity and Innovation Efficiency}

The conditional efficiency analysis reveals that both collaboration intensities, REGION and INTER, are significantly related to regional innovation efficiency with $p$-values of 0.006 and 0.01 , respectively. Figure 7 shows the non-parametrically estimated relationships for the two variables in a three-dimensional plot.

The two variables' relationships with the efficiency estimate are best described by an inverted-U shape. For most parts, the shape is very robust as the narrow bootstrapped error bands highlight (see Figure 8). However, very wide error bands prevent an interpretation of the curve for values of INTER below 0.1 and above $1.5 .^{19}$

Figures 9 and 10 provide detailed views on the results for REGION and INTER, showing the (non-parametrically fitted) trend lines for the third of the regions with the lowest (dashed), medium (solid) and highest (dotted) values of INTER and REGION, respectively.

Despite the considered time lag between R\&D employees and patent-based measures, the study is of cross-sectional nature. It means that the results have to be interpreted with care when making inference about the causal direction between collaboration intensity and regional innovation efficiency. Keeping this in mind, the analysis nevertheless shows that innovation efficiency and collaboration intensities are related at the regional level. The study thereby confirms firm-level findings by Arndt and Sternberg (2000), but contrasts results by Sternberg and Arndt (2001), Fritsch (2004) and Oerlemans and Meeus (2005).

The results clearly support hypothesis 1 : the relationship between the collaboration intensity among regional organizations and innovation performance of regions (as measured by innovation efficiency) shows as an inverted-U shape. Low and high collaboration intensities (strong deviation from the industry's average) are characteristics of innovation inefficient regions. The location of the curves' peaks, which are close to a value of one for REGION and INTER, suggests that collaboration intensity levels close to the industry's average are most frequently found among innovation efficient regions.

\footnotetext{
${ }^{18}$ The primary reason for the little change in the ranking is the heavily skewed regional distribution of patents. It implies a scarcity of comparison observations for regions with large patent numbers resulting in these being automatically deemed efficient.

${ }^{19}$ The analysis has been repeated for regions with at least 5,10 and 20 patents as well as under consideration of a dummy for East Germany ( $p$-values: REGION, 0.018; INTER, 0.003; EAST, 0.001). The results remain more or less the same as reported above. An exception being the negative trend for INTER, which is becoming less pronounced when small regions are not considered or it is controlled for regions being located in East Germany (which tend to be small in terms of patent output). See Figures 11 and 12.
} 


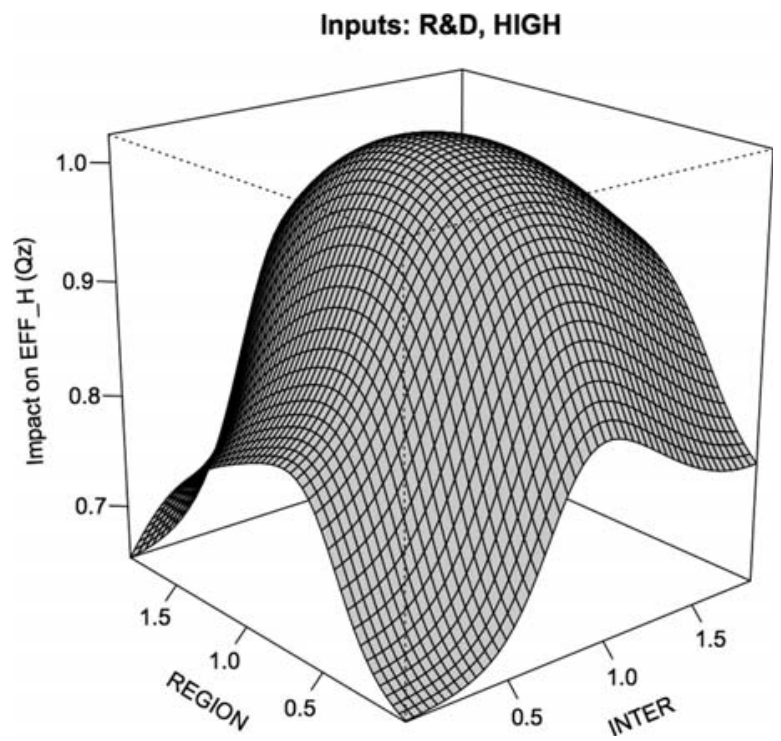

Figure 7. Relationship between EFF_H and REGION as well as INTER in three dimensions

In contrast, I have to reject hypothesis 2 , according to which high intensities of interregional collaboration relate to high regional innovation efficiency. Rather the contrary seems to hold. Intensity levels above the industry's average characterize regions that are innovation inefficient. This holds irrespectively of the intensity of regional collaboration (see Figure 10).

The findings moreover imply that hypothesis 4 has to be rejected as well. Regions with average (but balanced) intensities of regional and inter-regional collaboration outperform those with excessively high intensities. While regional and inter-regional collaboration have received much attention in the literature (see, e.g. Camagni, 1991), potential negative

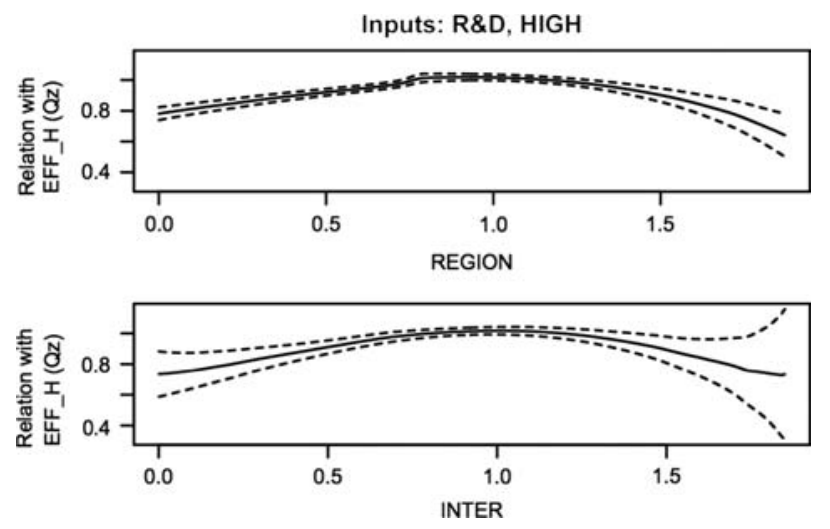

Figure 8. Results with error bands 


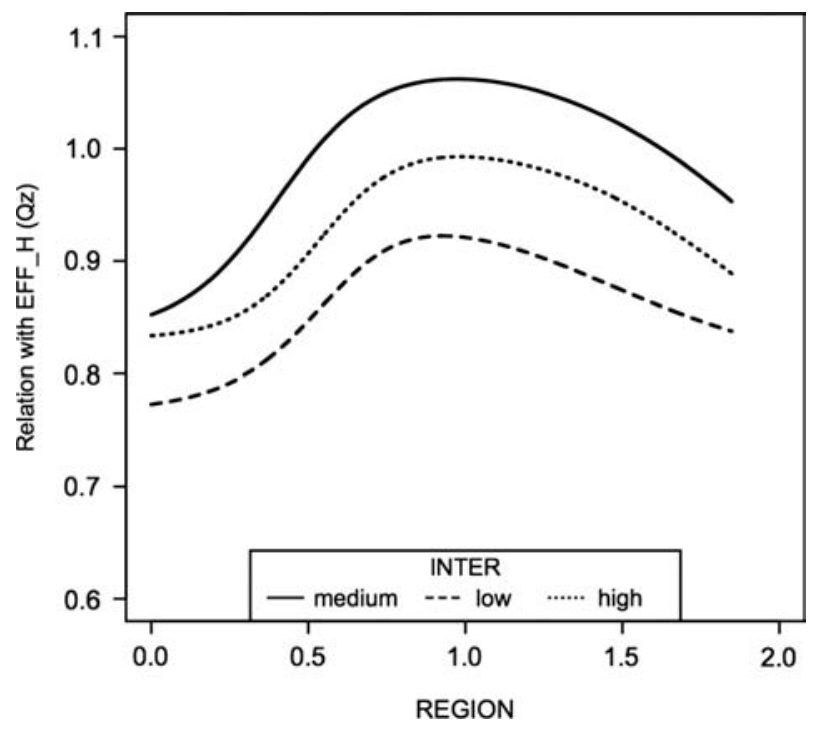

Figure 9. EFF_H relation with REGION

Inputs: R\&D, HIGH

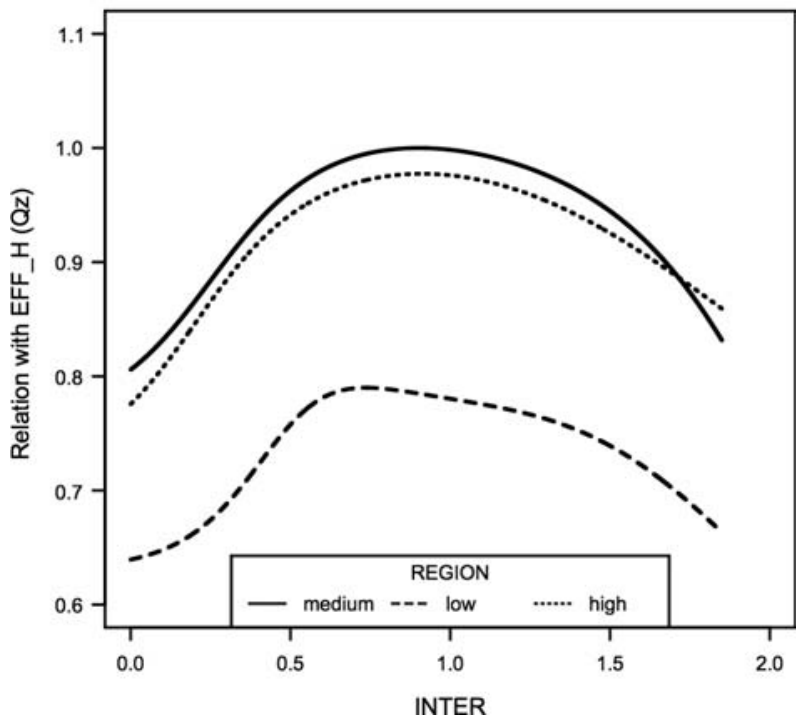

Figure 10. EFF_H relation with INTER

effects associated with too intense collaboration are less discussed. Porter (1990) suggests that intense collaboration can indicate a lack of rivalry, which in turn indicates a missing of incentives for innovating. Bathelt et al., (2004) furthermore suggest the possibility of "buzz congestion", which is caused by information overload. This means that as a result of very 

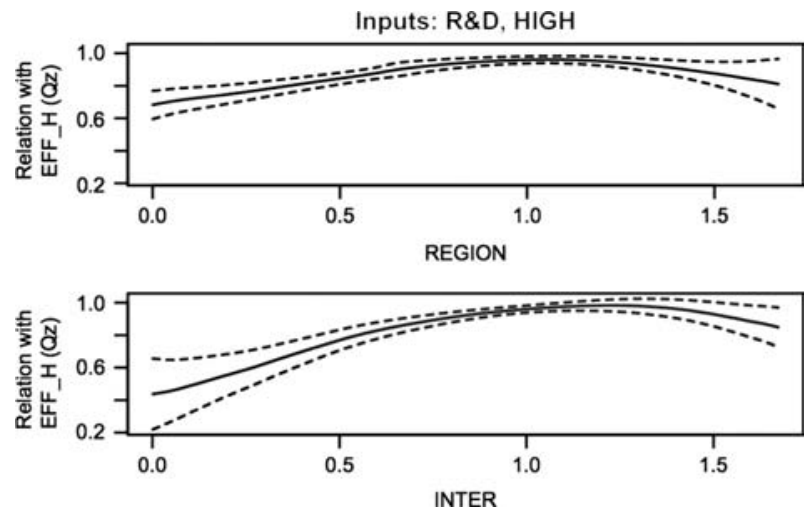

Figure 11. Results for regions with at least 10 patents

intense regional interactions, organizations are exposed to too much information and become unable to separate useful from useless information.

It has to be pointed out though that regions with below average values in REGION and INTER are more inefficient than those with above average intensities: the median efficiency for the third of the regions with the lowest values of REGION is 5.83, while the median efficiency of the third of the regions with the highest values is 2.33. The same numbers for INTER are 7.49 and 3.09, respectively. Accordingly, regions characterized by very high

Inputs: R\&D,HIGH
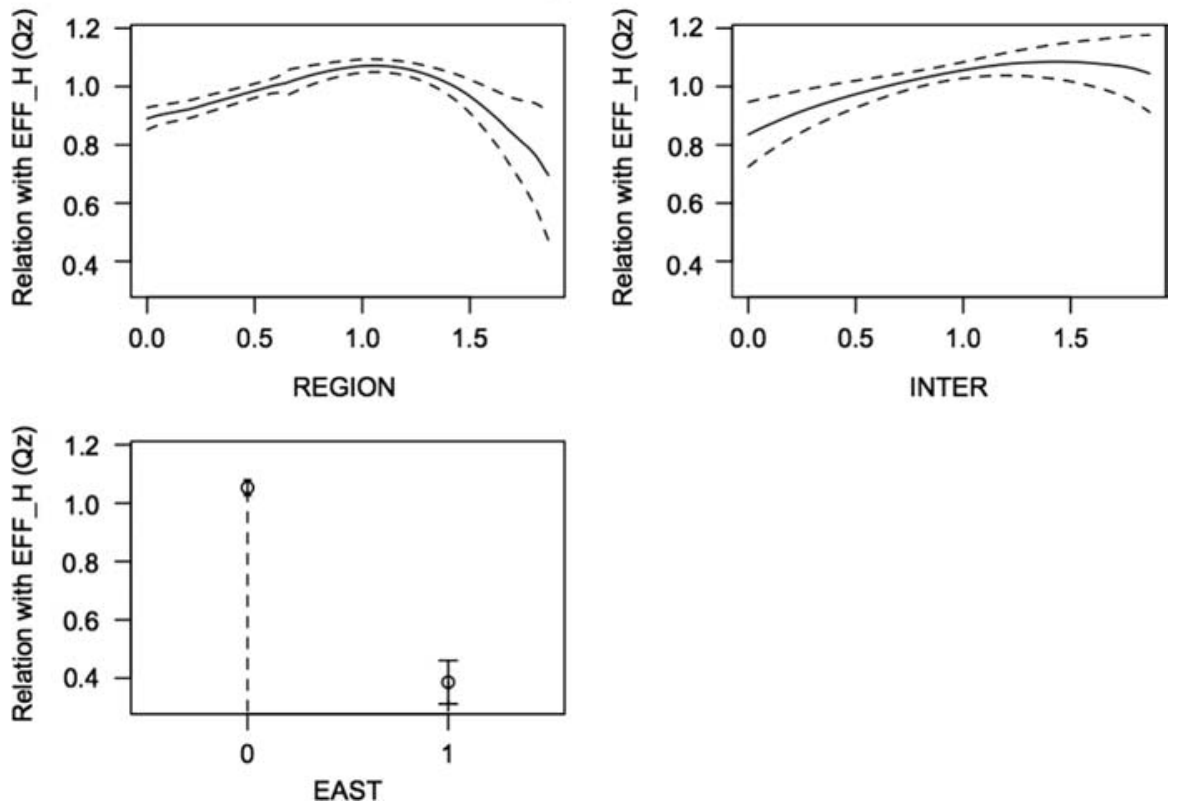

Figure 12. Results including dummy for East German regions 
collaboration intensities are more efficient than regions with very low intensities. Possibly this indicates that the negative effects related to very intense collaboration are less severe than those associated with little collaborating. This marks an interesting finding that clearly deserves future research.

Somewhat clearer are the explanations for why a misbalance in the geographic reach of organizations' collaborations can result in lower innovation efficiency. In line with hypothesis $\mathbf{3}$ are average levels and a balance of regional and inter-regional collaboration are found to characterize the most innovation efficient regions. Organizations in these regions exploit regional collaboration by showing considerable participation in regional networks (and potentially develop "local buzz"). Thereby, they benefit from the advantages of geographic proximity for collaboration. At the same time, they have sufficient access to inter-regional networks, which might be part of "global pipelines" of knowledge. This allows for overcoming "identified shortcomings in the local knowledge base" (Bathelt et al., 2004: 44).

The analysis furthermore provides empirical evidence for the existence of excessive regional and inter-regional collaboration situations that go hand in hand with lower innovation performance. Such situations of extremely unbalanced collaboration behavior are particularly relevant for small rural regions (those with low patent numbers) among which they are more frequently found to exist. This adds to the study of Mayer-Krahmer (1985) who finds that firms in regions with few potential cooperation partners (as it is usually the case in rural areas) perceived the shortage of knowledge in their region as "locational disadvantage" (531). For this reason, they are forced to engage more extensively in external collaboration. However, these lack the benefits of geographical proximity and thereby do not stimulate their innovation performance.

Mayer-Krahmer (1985) also argues that a firm's location inside or outside an agglomeration can be an important factor influencing its collaboration behavior. For the present study, this means that the availability of regional collaboration opportunities may induce a particular collaboration behavior. While I control for this "collaboration potential" effect in the empirical assessment (see Section 4.3), it might be the case that regions' levels of innovation efficiency impact the collaboration activities of their organizations (reversed causality). For instance, being highly innovative (which tends to go hand in hand with being innovation efficient) makes regions attractive partners for collaboration implying that these regions can be expected to show above average collaboration intensities. This is not supported by the empirical results since innovation efficient regions rather tend to show average and balanced collaboration intensities. However, I cannot rule out this issue of reversed causality as it could be argued that organizations in these regions have better capabilities balancing their collaboration activities. Given their attractiveness as collaboration partners, they might also be more discriminating in their choice of collaboration partners. Further research is needed to shed more light on this issue.

Table 6 summarizes the empirical findings with respect to the four hypotheses.

\section{Discussion and Conclusion}

The paper contributed to the literature by providing a detailed discussion on the relationship between regional levels of collaboration intensity and region's innovation performance. Moreover, this relation was quantitatively empirically analyzed utilizing data for all 270 German labor market regions and the Electrics \& Electronics industry. It thereby 
Table 6. Relationship between innovation efficiency and collaboration intensity

\begin{tabular}{|c|c|c|c|c|}
\hline & & \multicolumn{3}{|c|}{$\begin{array}{c}\text { Regional } \\
\text { collaboration intensity }\end{array}$} \\
\hline & & Low & Average & High \\
\hline \multirow[t]{6}{*}{$\begin{array}{l}\text { Inter-regional collaboration } \\
\quad \text { intensity }\end{array}$} & Low & Isolation & A & $\begin{array}{r}\text { Excessive regional } \\
\text { collaboration }\end{array}$ \\
\hline & & $(-)$ & $(+)$ & $(-)$ \\
\hline & Average & $B$ & Balanced coop & C \\
\hline & & $(-)$ & $(++)$ & $(+)$ \\
\hline & High & $\begin{array}{l}\text { Excessive inter-regional } \\
\text { collaboration }\end{array}$ & $\mathrm{D}$ & Excessive collaboration \\
\hline & & $(-)$ & $(+)$ & $(-)$ \\
\hline
\end{tabular}

$(+)$ indicates a positive and $(-)$ a negative relationship with regional innovation efficiency.

extended previous research that either applies a qualitative approach (e.g. Asheim and Isaksen, 2002) or covers only a small number of regions (e.g. Fritsch, 2004). In addition, a novel and fully non-parametric approach was chosen to estimate regional innovation efficiency as well as for analyzing its relation with collaboration intensity.

It was shown that this relationship is empirically significant and shows an inverted-U shape. More precisely, the intensities of regional and inter-regional collaboration close to the industry's average characterize innovation efficient regions. In contrast, regions with extremely high or low, as well as unbalanced regional and inter-regional collaboration intensities are more frequently found among innovation inefficient regions.

The study has a number of shortcomings that may lead the track for future research. First of all, the study relies on patent data to approximate innovation as well as collaboration behavior. In particular, the latter is not unproblematic as there are many unobservable factors that influence firms' engagement in collaboration and in collaborative patenting (see, e.g. Giuri and Mariani, 2005). The use of patent data also implied that collaboration intensity was purely quantitatively defined, that is, differences in the quality of collaboration were not taken into account. However, the complementarity of partners is crucial for successful collaboration (Cantner and Meder, 2007). Alternative collaboration measures should therefore be employed in future research.

The availability of data also restricted the analysis to a cross-sectional approach that makes it more problematic to infer about the causal relationship between collaboration intensity and regional innovation performance. Longitudinal studies might also be more interesting as they can help to investigate the potentially varying importance of regional and inter-regional collaboration intensities over industries' life cycles (see, e.g. Neffke et al., 2010).

Another shortcoming concerns the paper's focus on a single industry. Hence, the findings remain restricted in their generality, as the observed patterns might be a characteristic of this particular industry.

Despite these limitations, the study gives some indication for the design of regional policy. In line with most of the literature, it is shown that collaboration is often positively related to innovation. This means that policy programs supporting collaboration, network 
formation and joint $R \& D$ projects are useful approaches for supporting innovation activities (see on this also Beers et al., 2008; Fornahl et al., 2011).

Some policy initiatives, like the BioRegio, InnoRegio or InnoNet in Germany, are however designed to stimulate collaboration among organizations within a particular region (see Eickelpasch and Fritsch, 2005). The present study provides empirical evidence for the existence of an optimal level of regional collaboration intensity. This level marks a crucial threshold for the application of such collaboration stimulating policies, which are consequently justified only in situations of regional collaboration intensity being below this threshold. Or, in other words, if the regional collaboration intensity exceeds this threshold, policies aiming at further stimulating collaboration can induce situations of excessive regional collaboration that may even hamper innovation. Accordingly, the existence of such a threshold should be considered when designing this type of innovation support initiative. While the empirical identification of this threshold is surely challenging, it has to be pointed out that the negative effects on innovation are primarily related to extreme situations of excessive collaboration. These are characterized by a severe deviation from the average collaboration intensity and hence they can be detected more easily.

Another issue that has to be taken into account when designing policies that support collaborative innovation is the complementarity of regional and inter-regional collaboration. For instance, if collaboration among regional organizations is to be supported, it is important to consider their intensity of inter-regional collaboration as well. The findings in this paper clearly show that the positive effects associated with regional collaboration are conditional on organizations being also well embedded into inter-regional knowledge relations. Accordingly, the study highlights that supporting collaboration with a particular geographical focus requires the identification of the type of collaboration (regional or inter-regional) that is actually underdeveloped.

\section{References}

Arndt, O. and Sternberg, R. (2000) Do manufacturing firms profit from intraregional innovation linkages? An empirical based answer, European Planning Studies, 8(4), pp. 465-485.

Arundel, A. and Kabla, I. (1998) What percentage of innovations are patented? Empirical estimates for European firms, Research Policy, 27(2), pp. 127-141.

Asheim, B. T. and Isaksen, A. (2002) Regional innovation systems: the integration of local "sticky" and global "ubiquitous" knowledge, Journal of Technology Transfer, 27, pp. 77-86.

Bathelt, H., Malmberg, A. and Maskell, P. (2004) Clusters and knowledge: local buzz, global pipelines and the process of knowledge creation, Progress in Human Geography, 28(1), pp. 31-56.

Beers, C., Berghäll, E. and Poot, T. (2008) R\&D internationalization, R\&D collaboration and public knowledge institutions in small economies: evidence from Finland and the Netherlands, Research Policy, 39(2), pp. 294-308.

Boschma, R. A. (2005) Proximity and innovation: a critical assessment, Regional Studies, 39(1), pp. 61-74.

Brenner, T. and Broekel, T. (2011) Methodological issues in measuring innovation performance of spatial units, Industry and Innovation, 18(1), pp. 7-37.

Broekel, T. (2007) A concordance between industries and technologies—-matching the technological fields of the Patentatlas to the German industry classification. Jenaer Economic Research Papers 2007-013.

Broekel, T. and Binder, M. (2007) The regional dimension of knowledge transfers-a behavioral approach, Industry and Innovation, 14(2), pp. 151-175. 
Broekel, T. and Brenner, T. (2007) Measuring regional innovativeness—a methodological discussion and an application to one German industry. DIME Working Paper 2007-13.

Cainelli, G., Mancinelli, S. and Mazzanti, M. (2007) Social capital and innovation dynamics in district-based local systems, The Journal of Socio-Economics, 36(6), pp. 932-948.

Camagni, R. (1991) Local "milieu", uncertainty and innovation networks: towards a new dynamic theory of economic space, in: R. Camagni (Ed.), Innovation Networks: Spatial Perspectives (London and New York: Belhaven Press).

Cantner, U. and Meder, A. (2007) Technological proximity and the choice of cooperation partners, Journal of Economic Interaction and Coordination, 2, pp. 45-65.

Cantner, U. and Meder, A. (2008) Regional and technological effects of cooperation behavior. Jenaer Economic Research Papers 14.

Cassiman, B. and Veugelers, R. (2002) R\&D cooperation and spillovers: some empirical evidence from Belgium, American Economic Review, 92, pp. 1169-1184.

Cazals, C., Florens, J. -P. and Simar, L. (2002) Nonparametric frontier estimation: a robust approach, Journal of Econometrics, 106(1), pp. $1-25$.

Coelli, T., Prasada Rao, D. S. and Battese, G. (1998) An Introduction to Efficiency and Productivity Analysis (Boston: Kluwer Academic).

Daraio, C. and Simar, L. (2007) Advanced Robust and Nonparametric Methods in Efficiency Analysis-Methodology and Applications (Boston: Kluwer Academic).

De Bondt, R., Slaets, P. and Cassiman, B. (1992) The degree of spillovers and the number of rivals for maximum effective R\&D, International Journal of Industrial Organization, 10, pp. 35-54.

De Witte, K. and Kortelainen, M. (2009) Blaming the exogenous environment?. Conditional efficiency estimation with continuous and discrete exogenous variables, MPRA Paper 14034.

Eickelpasch, A. and Fritsch, M. (2005) Contests for cooperation-a new approach in German innovation policy, Research Policy, 34, pp. 1269-1282.

Faggian, A. and McCann, P. (2006) Human capital flows and regional knowledge assets: a simultaneous equation approach, Oxford Economic Papers, 52, pp. 475-500.

Feldman, M. P. and Florida, R. (1994) The geographic sources of innovation: technological infrastructure and product innovation in the United States, Annals of the Association of American Geographers, 84(2), pp. 210-229.

Fornahl, D., Broekel, T. and Boschma, R. A. (2011) What drives patent performance of German biotech firms? The impact of R\&D subsidies, knowledge networks and their location, Papers in Regional Science, 90(2), pp. 395-418.

Fritsch, M. (2000) Interregional differences in R\&D activities—an empirical investigation, European Planning Studies, 8(4), pp. 409-427.

Fritsch, M. (2003) How and why does the efficiency of regional innovation systems differ, in: J. Brocker, D. Dohse \& R. Soltwedel (Eds), Innovation Clusters and Interregional Competition (Berlin: Springer-Verlag).

Fritsch, M. (2004) Cooperation and the efficiency of regional R\&D activities, Cambridge Journal of Economics, 28, pp. 829-846.

Fritsch, M. and Franke, G. (2004) Innovation, regional knowledge spillovers and R\&D cooperation, Research Policy, 33, pp. $245-255$.

Fritsch, M. and Slavtchev, V. (2008) Determinants of the efficiency of regional innovation systems, Regional Studies. doi: $101080 / 00343400802251494$.

Giuri, P. and Mariani, M. (2005) Everything you always wanted to know about inventors (but never asked): evidence from the Patval-EU survey, LEM Working Paper Series 2020.

Grabher, G. (1993) The weakness of strong ties: the lock-in of regional development in the Ruhr area, in: G. Grabher (Ed.), The Embedded Firm-On the Socioeconomics of Industrial Networks, pp. 255-277 (London and New York: Routledge), Reprinted in 1994.

Graf, H. (2010) Gatekeepers in regional networks of innovation, Cambridge Journal of Economics. doi: 101093/cje/beq001.

Greif, S. and Schmiedl, D. (2002) Patentatlas 2002 Dynamik und Strukturen der Erfindungstätigkeit. Deutsches Patent- und Markenamt, München.

Greif, S., Schmiedl, D. and Niedermeyer, G. (2006) Patentatlas 2006. Regionaldaten der Erfindungstätigkeit. Deutsches Patent- und Markenamt, München.

Griliches, Z. (1979) Issues in assessing the contribution of R\&D to productivity growth, Bell Journal of Economics, 10, pp. 92-116.

Griliches, Z. (1990) Patent statistics as economic indicators: a survey, Journal of Economic Literature, 28, pp. 1661-1701.

Hagedoorn, J. (2003) Sharing intellectual property rights-an exploratory study of joint patenting amongst companies, Industrial and Corporate Change, 12(5), pp. 1035-1050.

Isaksen, A. (2001) Building regional innovation systems: is endogenous industrial development possible in the global economy?, Canadian Journal of Regional Science, 24(1), pp. 101-120. 
Jaffe, A. (1989) Real effects of academic research, American Economic Review, 79(5), pp. 957-970.

Kesteloot, K. and Veugelers, R. (1995) Stable R\&D cooperation with spillovers, Journal of Economics and Management, 4, pp. 651-672. Legler, H., Licht, G. and Egeln, J. (2001) Zur Technologischen Leistungsfähigkeit Deutschlands—Zusammenfassender Endbericht 2000. Gutachten im Auftrag des Bundesministeriums für Bildung und Forschung.

Meyer-Krahmer, F. (1985) Innovation behaviour and regional indigenous potential, Regional Studies, 19(6), pp. 523-534.

Neffke, F., Henning, M. S., Boschma, R. A., Lundquist, K. -J. and Olander, L. -O. (2010) The dynamics of agglomeration externalities along the life-cycle of industries, Regional Studies, 45(1), pp. 49-65.

Oerlemans, L. A. G. and Meeus, M. T. H. (2005) Do organizational and spatial proximity impact on firm performance, Regional Studies, 39(1), pp. 89-104.

Porter, M. (1990) The Competitive Advantage of Nations (New York: Free Press).

Powell, W. W., Walter, W., Koput, K. W. and Smith-Doerr, L. (1996) Inter-organizational collaboration and the locus of innovation: networks of learning in biotechnology, Administrative Science Quarterly, 41(1), pp. 116-145.

Saxenian, A. (1998) Regional Advantage-Culture and Competition in Silicon Valley and Route 128 (Cambridge, MA: Harvard University Press).

Schmoch, U., Laville, F., Patel, P. and Frietsch, R. (2003) Linking technology areas to industrial sectors, Final report to the European Commission, DG Research, Brussels.

Simar, L. and Wilson, P. W. (2007) Estimation and inference in two-stage semi-parametric models of production processes, Journal of Econometrics, 136, pp. 31-64.

Sternberg, R. (1999) Innovative linkages and proximity: empirical results from recent surveys of small and medium sized firms in German regions, Regional Studies, 33(6), pp. 529-540.

Sternberg, R. and Arndt, O. (2001) The firm or the region: what determines the innovation behavior of European firms?, Economic Geography, 77(4), pp. 364-382.

Storper, M. and Venables, A. J. (2004) Buzz: face-to-face contact and the urban economy, Journal of Economic Geography, 4, pp. $351-370$.

Teece, D. (1986) Profiting from technological innovation: implication for integration, collaboration, licensing and public policy, Research Policy, 15, pp. 285-305.

Uzzi, B. (1996) The sources and consequences of embeddedness for the economic performance of organizations: the network effect, American Sociological Review, 61(4), pp. 674-698.

Uzzi, B. (1997) Social structure and competition in interfirm networks: the paradox of embeddedness, Administrative Science Quarterly, 42(1), pp. 35-67. 